\title{
NON-AMPHICHEIRALITY OF THE SPECIAL ALTERNATING LINKS
}

\author{
KUNIO MURASUGI ${ }^{1}$
}

Introduction. An oriented (polygonal) $\operatorname{link}^{2} k$ is said to be $a m p h i$ cheiral if it is isotopic to its mirror imaged link. Our study started in the direction of proving the following conjecture.

CONJECTURE. All special alternating ${ }^{3}$ links are not amphicheiral.

As is well known, all torus knots are not amphicheiral. Any torus link that is alternating and prime is an elementary torus link, i.e. a torus link of type $(n, 2)$, and any prime special alternating link for which $\Delta(0)=1$ is an elementary torus link, where $\Delta(t)$ denotes the $A$ polynomial $^{4}$ of a link. In this paper, the following will be proved.

Theorem A. A special alternating link for which $\Delta(0)=2$ is not amphicheiral.

Theorem B. A special alternating knot of odd genus is not amphicheiral.

1. Let $\kappa$ be a link type and let $k$ be an oriented link representing $\kappa$. Let $K$ be a (normed regular) projection of $k$ onto $S^{2} C S^{3}[8] . K$ is oriented by the orientation induced by that of $k$. Let $N(K)$ denote the number of crossing points in $K$ and let $N(\kappa)$ denote the minimum of the crossing points in projections of any link representing $\kappa . N(\kappa)$ is a well-known link invariant. A projection $K$ is said to be reduced if $N(K)=N(\kappa)$. Then we have the following classical theorem $[2 ; 4]$.

THEOREM 1.1. If a reduced projection of $k$ is special alternating, then $k$ is not amphicheiral.

Although every link possesses at least one special projection, there exist alternating links that have no special alternating projection [5]. In the following by the projection of an alternating, or special alternating, link will be meant an alternating, or special alternating, projection.

Since the product of the second torsion numbers of a knot is equal

Received by the editors September 9, 1961.

1 The kind suggestions of the referee are gratefully acknowledged.

${ }^{2}$ A link of multiplicity $\mu(\geqq 1)$ is the union of $\mu$ ordered, pairwise disjoint simple closed curves in 3-sphere $S^{3}$. A knot is a link of multiplicity one.

${ }^{8}$ For the definition, see [5, p. 278].

4 For the definition, see [5, p. 280 and (1.11)]. We may assume without loss of generality that the leading coefficient of $\Delta(t)$, i.e. $\Delta(0)$, is greater than zero. 
to $|\Delta(-1)|$, Goeritz's Theorem [8, p. 30] simply implies the following

THEOREM 1.2. If $|\Delta(-1)| \equiv 3(\bmod 4)$ then the knot is not amphicheiral.

2. Theorem $B$ will be shown from Theorem 1.2 and the following

Lемма 2.1. If $k$ is a special alternating knot of genus $m$, then $|\Delta(-1)| \equiv 2 m+1(\bmod 4)$.

Proof. The $A$-polynomial of $k$ is of the form $[1 ; 5]$ :

$$
\Delta(t)=a_{0}-a_{1} t+a_{2} t^{2}+\cdots+(-1)^{i} a_{i} t^{i}+\cdots+a_{2 m} t^{2 m},
$$

where $a_{0}, a_{1}, \cdots, a_{2 m}>0$. Then we have $\Delta(-1)=\sum_{i=0}^{2 m} a_{i}>0$. Since $a_{j}=a_{2 m-j}$, for $j=0,1, \cdots, m$, it follows that $\Delta(-1)$ $=2\left(a_{0}+a_{1}+\cdots+a_{m-1}\right)+a_{m}$. On the other hand, since $\Delta(1)$ $=\sum_{i=0}^{2 m}(-1){ }^{i} a_{i}=1[5]$, we have

$$
\begin{aligned}
\Delta(-1)= & 2\left(a_{0}+a_{1}+\cdots+a_{m-1}\right) \\
& +(-1)^{m}\left\{1-2\left[a_{0}-a_{1}+\cdots+(-1)^{m-1} a_{m-1}\right]\right\} \\
= & 2\left\{\left[1+(-1)^{m+1}\right] a_{0}+\left[1+(-1)^{m+2}\right] a_{1}+\cdots\right. \\
& \left.+\left[1+(-1)^{2 m}\right] a_{m-1}\right\}+(-1)^{m} \\
\equiv & (-1)^{m} \equiv 1+2 m(\bmod 4) .
\end{aligned}
$$

3. Let $G$ be a (finite planar), directed or undirected, graph. A sequence $P=\left(a_{0}, A_{1}, a_{1}, \cdots, A_{n}, a_{n}\right), a_{i}$ and $A_{j}$ denoting vertices and edges, is an undirected path in $G$ from $a_{0}$ to $a_{n}$ if $a_{0}, a_{1}, \cdots, a_{n-1}$ are all distinct and if $a_{j-1}$ and $a_{j}$ are two ends in $G$ of $A_{j}$ for $j=1,2, \cdots, n$. Specially a path $P$ is said to be directed from $a_{0}$ to $a_{n}$ if $A_{j}$ are directed from $a_{j-1}$ to $a_{j}$. A path is called a circuit (or $n$-circuit) if $a_{0}=a_{n}$. The valency of a vertex is defined as the number of edges in $G$ incident to it.

Now let $K$ be a projection of a link $k$. Then we can uniquely define a graph $G(K)$ and a dual graph $G^{*}(K)$ of $K$ [5]. To each crossing in $K$ there correspond a unique edge of $G(K)$ and a unique edge of $G^{*}(K)$. Each edge of $G(K)$ is directed in such a way that it crosses the overpassing segment from left to right at the crossing point corresponding to it. Thus $G(K)$ and $G^{*}(K)$ are directed. If $k$ is a special alternating link, then we can choose the graph $G(K)$ in such a way that the valency of each vertex of $G(K)$ is even and $G(K)$ is the union of directed circuits.

Conversely given an undirected graph $G$, there are exactly two alternating projections. One is the projection of the mirror imaged 
link of the other. If they are oriented, then $G$ is directed. Since the $A$-polynomial of a mirror imaged link coincides with that of an original link, the $A$-polynomial of the alternating link induced from a given graph $G$ is uniquely determined. Thus we can call it the $A$ polynomial of $G$.

We can assume, hereafter, that $G(K)$ and $G^{*}(K)$ are connected and contain no 1-circuit.

A projection $K$ is of the bridge form of type $\left(p_{1}, p_{2}, \cdots, p_{r}\right)$ if $G(K)$ or $G^{*}(K)$ consists of $r$ paths $P_{1}, P_{2}, \cdots, P_{r}$ from a vertex $u$ to another vertex $v$, having no common vertices except $u$ and $v$, where $P_{i}$ contain $p_{i}$ vertices other than $u$ and $v . k$ is called, then, a bridge link of type $\left(p_{1}, p_{2}, \cdots, p_{r}\right)$. If $k$ is a special alternating bridge link of type $\left(p_{1}, p_{2}, \cdots, p_{r}\right)$, then in $G(K), r$ is always even, and one half of $r$ paths are directed from $u$ to $v$ and the other half are directed reversely. The number of vertices of $G(K)$ equals $^{5} d(K)+1$. For example, the special alternating bridge link of type $(p, q)$ coincides with the torus link of type $(p+q+2,2)$. Moreover, a special alternating knot of genus one is an alternating bridge knot of type $(p, q, r)$.

4. To characterize the links for which $\Delta(0)=2$, we need the following

LEMMA 4.1. Let $G$ be the directed graph of a projection $K$ of a special alternating link and let $G^{\prime}$ be the directed graph obtained from $G$ by removing a directed circuit $C$. Denoting the $A$-polynomials of $G$ and $G^{\prime}$ by $\Delta(t)$ and $\Delta^{\prime}(t)$ respectively, we have

$$
\Delta(0) \geqq \Delta^{\prime}(0)+2^{n-1}-1,
$$

where $n$ denotes the number of vertices in $C \cap G^{\prime}$.

Proof. Let us denote $I_{p, q}(i, j)$ the $p \times q$ matrix whose elements are all zero except the $(i, j)$ element, which is equal to one. By definition, then, knot matrices ${ }^{6} M, M^{\prime}$ of $G, G^{\prime}$ are of the forms:

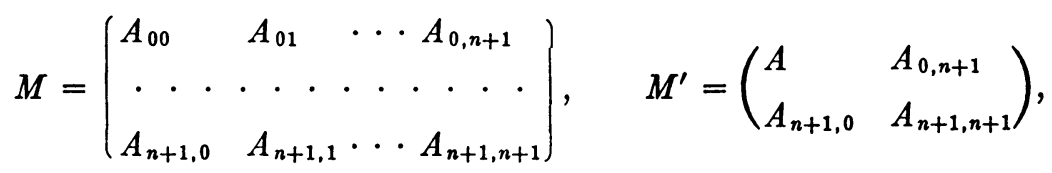

where $A_{0 i}=-I_{n, p_{i}}(i, 1), A_{j, 0}=-I_{p_{j}, n}\left(p_{j}, j+1\right), A_{n, 0}=-I_{p_{n}, n}\left(p_{n}, 1\right)$,

- $d(k)$ or $d(K)$ denotes the degree of the $A$-polynomial $\Delta(t)$ of $k$.

- By the knot matrix of $G$ is meant the knot matrix of the alternating link induced by $G$. For the definition and properties of a knot matrix, and for the matrix notations, see $[5 ; 6]$. 


$$
\begin{gathered}
A_{k k}=I-\sum_{i=1}^{p_{k}-1} I_{p_{k}, p_{k}}(i, i+1), \\
\left(i=1, \cdots, n, j=1, \cdots, n-1, k=1, \cdots, n, p_{l} \geqq 0 \text { for all } l\right),
\end{gathered}
$$

$A=A_{00}-I, I$ denoting the identity matrix, and $A_{i j}=0$ $(i, j=1, \cdots, n+1, i \neq j) . A_{00}$ and $A$ correspond to $n$ common vertices in $C \cap G^{\prime}$. Since $\Delta(0)=\operatorname{det} \tilde{M}(n, n)$ and $\Delta^{\prime}(0)=\operatorname{det} \tilde{M}^{\prime}(n, n)$ by definition, it follows easily that

$$
\Delta(0)=\Delta^{\prime}(0)+\sum_{\substack{1 \leq i_{1}<i_{2}<\cdots<i_{\lambda} \leq n-1 \\ \lambda=1,2, \cdots, n-1}} \operatorname{det} N_{i_{1} i_{2} \cdots i_{\lambda}} ;
$$

$N_{i_{1} i_{2} \ldots i_{\lambda}}$ is the matrix obtained from $\tilde{M}^{\prime}(n, n)$ by replacing the $i_{j}$-row $(1 \leqq j \leqq \lambda)$ by $R_{i_{j}}=I_{1, n+8-1}\left(1, i_{j}\right)-I_{1, n+8-1}\left(1, i_{j}+1\right)$, where $s$ denotes the number of columns of $A_{0, n+1}$ and we define $R_{n-1}$ $=I_{1, n+s-1}(1, n-1)$. To prove $(4.1)$ it is sufficient to show that $\operatorname{det} N_{i_{1} i_{2}} \ldots i_{\lambda}>0$. This is obvious from Lemma 2.4 (i) in [5], since $N_{i_{1}} \ldots i_{\lambda}$ is of strongly special type on the row. ${ }^{7}$ q.e.d.

If $n=2$, the Lemma 4.1 is sharpened as follows.

Lemma 4.2. $\Delta(0)=\Delta^{\prime}(0)+\Delta^{\prime \prime}(0)$, where $\Delta^{\prime \prime}(t)$ denotes the $A$-polynomial of the graph obtained from $G^{\prime}$ by identifying two common vertices.

By using Lemma 4.1, we can complete the characterization. In fact, we have

LemMa 4.3. A prime special alternating link $k$ for which $\Delta(0)=2$ is a special alternating bridge link of type $(p, q, r, s)$, where $p+q+r+s+1$ $=d(k)$, and conversely.

Proof. Let $K$ be a projection of $k$ and let $G^{\prime}$ be the graph obtained in Lemma 4.1. Then (4.1) shows that $2=\Delta(0) \geqq \Delta^{\prime}(0)+2^{n-1}-1$. This implies that $\Delta^{\prime}(0)=1$ and $n=2$, since $k$ is prime. Thus $K$ is of the form as is required in the lemma. It is obvious that $p+q+r+s+1$ $=d(k)$. Converse is clear.

5. To prove Theorem A, some lemmas on $N(\kappa)$ will be required. In Lemmas 5.1-5.3, $k$ need not be alternating.

Lemma 5.1. For any nontrivial link $k, N(\kappa) \geqq d(k)+1$. Equality holds if and only if $k$ is an elementary torus link.

This is obvious from $[1 ; 5]$.

LEMma 5.2. For any special projection $K$ of any link $k$ for which $\Delta(0)=2, N(K) \geqq d(k)+3$.

7 For the definition, see [5, p. 289]. 
Proof. From Lemma 5.1, we see that $G(K)$ contains at least $d(k)+1$ edges. Since $K$ is special, the valencies of all vertices must be even. If the valency of each vertex is two, then $G$ is a circuit, since $G$ is connected. Thus we have $\Delta(0)=1$, which is a contradiction. If only one vertex is of valency four, then $G$ consists of two circuits having one vertex in common, from which it follows that $\Delta(0)=1$. Thus $G$ contains at least two vertices of valency $\geqq 4$. It follows that $N(K) \geqq d(K)+3$.

Lemma 5.3. For any nonspecial projection $K$ of any link, $N(K)$ $>d(K)+3$.

Proof. We can define a matrix for any link $[5 ; 6]$. Then Corollary 1.40 in [5] can be extended as follows. Let $K=K_{1} * K_{2} * \cdots * K_{p}$ $(p \geqq 2),{ }^{8}$ and let $\Delta_{i}(t)$ denote the $A$-polynomials of $K_{i}$. Then we have

$$
\Delta(0)=\prod_{i=1}^{p} \Delta_{i}(0) \quad \text { and } \quad d(K)=\sum_{i=1}^{p} d\left(K_{i}\right)
$$

Thus all $\Delta_{i}(0)$ except one, say $\Delta_{p}(0)$, are equal to one, and $\Delta_{p}(0)=2$. Hence from Lemmas 5.1, 5.2 we have

$$
N\left(K_{i}\right) \geqq d\left(K_{i}\right)+1 \text { and } N\left(K_{p}\right) \geqq d\left(K_{p}\right)+3 .
$$

Consequently it follows from (5.1) and Lemma 3.29 in [5],

$$
\begin{aligned}
N(K) & =\sum_{i=1}^{p-1}\left(d\left(K_{i}\right)+1\right)+d\left(K_{p}\right)+3 \\
& =\sum_{i=1}^{p} d\left(K_{i}\right)+(p-1)+3 \\
& =d(K)+p+2>d(K)+3,
\end{aligned}
$$

q.e.d.

Now any special alternating bridge link $k$ of type $(p, q, r, s)$ possesses a projection $K$ with $d(K)+3$ crossing points, which is reduced and special alternating. Thus from Theorem 1.1 it follows that $k$ is not amphicheiral. Combining with Lemma 4.2 , we have Theorem A.

APPENDIX. Since a special alternating bridge link of type $(0,0, p, q)(p, q \geqq 0)$ is of two bridges, we can prove the non-amphicheirality of links of this kind by means of a theorem of $\mathrm{H}$. Schubert [9]. In fact, we see that $\alpha=2 p q+3 p+3 q+4$ and $\beta=2 p+3$ or $2 q+3$, where $(\alpha, \beta)$ denotes the normal form of this link $[7 ; 9]$. From this it is easy to show that $\beta^{2} \not \equiv-1(\bmod 2 \alpha)$.

8 For the definition, see $[5$, p. 293]. 


\section{REFERENCES}

1. R. H. Crowell, Genus of alternating link types, Ann. of Math. (2) 69 (1959), 258275.

2. A. Fischer, Gruppen und Verkettungen, Comment. Math. Helv. 2 (1930), 253268.

3. Y. Hashizume, On the uniqueness of the decomposition of a link, Osaka Math. J. 10 (1958), 283-300.

4. C. N. Little, Non alternate \pm knots, Trans. Roy. Soc. Edinburgh 39 (19), 771778.

5. K. Murasugi, On alternating knots, Osaka Math. J. 12 (1960), 277-303.

6. - On the definition of the knot matrix, Proc. Japan Acad. 37 (1961), 220221.

7. - Remarks on knots with two bridges, ibid. 294-297.

8. K. Reidemeister, Knotentheorie, Ergebnisse der Math. 11 (1932).

9. H. Schubert, Knoten mit zwei Brucken, Math. Z. 65 (1956), 133-170.

Hosei UNIVERSITY, JAPAN, AND

UNIVERSITY OF TORONTO 